EXTENDED REPORT

\title{
Systolodiastolic variations of blood flow during central retinal vein occlusion: exploration by dynamic angiography
}

\author{
M Paques, O Baillart, O Genevois, A Gaudric, B I Lévy, J Sahel
}

Br J Ophthalmol 2005;89:1036-1040. doi: 10.1136/bjo.2004.061275

See end of article for authors' affiliations

....................

Correspondence to: Michel Paques, MD, PhD, Department of

Ophthalmology, Fondation Ophtalmologique Rothschild, 25 rue Manin, 75019 Paris, France; michel.paques@laposte. net

Accepted for publication 1 January 2005

\begin{abstract}
Background/aim: In patients with acute central retinal vein occlusion (CRVO), dynamic angiography may reveal the presence of pulsatile flow (termed here pulsatile venular outflow, PVO) within first order veins (that is, the large veins). The main goal of this study was to investigate the mechanism underlying PVO. Methods: 10 patients with CRVO and PVO were included. Quantitative and qualitative analysis of venous flow on dynamic angiograms allowed the correlation, temporally, of second and first order vein flow on the one hand, and venous flow and systolic cycle on the other.

Results: Analysis of the time-velocity curve showed that (1) the onset of arterial systole preceded the onset of PVO by less than 0.08 seconds $(n=5)$; (2) PVO onset was simultaneous to the time of onset of minimal flow $\left(V_{\min }\right)$ in first order veins $(n=10)$; $(3)$ the time of onset of maximal flow $\left(V_{\text {max }}\right)$ in first order veins occurred 0.20-0.44 seconds after the onset of PVO $(n=6)$.

Conclusions: During CRVO with severe reduction in blood flow, the presence of PVO is the result of the existence of a distinct haemodynamic regimen in first and second order veins. These data support the hypothesis that second order veins flow is synchronous with the arterial flow, while the delayed peak flow in first order veins may reflect the consequences of the delayed IOP curve and/or of intermittent venous compression.
\end{abstract}

blood velocity. Moreover, arterial and venous flow can be simultaneously analysed.

In the present study, we analysed the temporal variations in venous flow in first and second order veins during CRVO. To achieve this, we analysed the dynamic angiogram in a series of patients in which the progression of PVO was clearly visible because of severe reduction in blood flow. Our results lead us to suggest that the visualisation of PVO was linked to the existence of distinct flow regimens in first and second order veins.

\section{PATIENTS AND METHODS}

Among a cohort of patients with CRVO included between July 2003 and May 2004 in a prospective internal review board approved study, the charts of 10 patients with clearly discernable PVO on dynamic angiography were selected for further analysis. All patients had a complete ophthalmological examination by one of us (MP), and gave informed consent at the time of inclusion.

\section{Dynamic angiography protocol}

Indocyanine green (ICG; Infracyanine, Société d'Etude et de Recherches Biologiques, Paris, France) dynamic angiography in the peripapillary area was recorded using either a confocal scanning laser ophthalmoscope (SLO; Heidelberg retina angiograph, Dossenheim, Germany) with the following capture parameters: field $20^{\circ}$; image frequency 20.6/s; definition $256 \times 256$ pixels, or a fundus video camera equipped with diode laser $(795 \mathrm{~nm}$ ) illumination (TRC 50 IAL, Topcon, Japan)..$^{10}$ The procedure in both cases comprised pupil dilation and injection into the antecubital vein of $5 \mathrm{mg}$ of ICG in $1 \mathrm{ml}$ of glucosed water by a $2.5 \mathrm{ml}$ syringe connected to a 23 gauge tubing, in less than 2 seconds. In addition, in two patients (cases 8 and 9), the Doppler signal

Abbreviations: AVFT, arteriovenous filling time; CRVO, central retinal vein occlusion; ICG, indocyanine green; PVO, pulsatile venular oufflow; SLO, scanning laser ophthalmoscope 

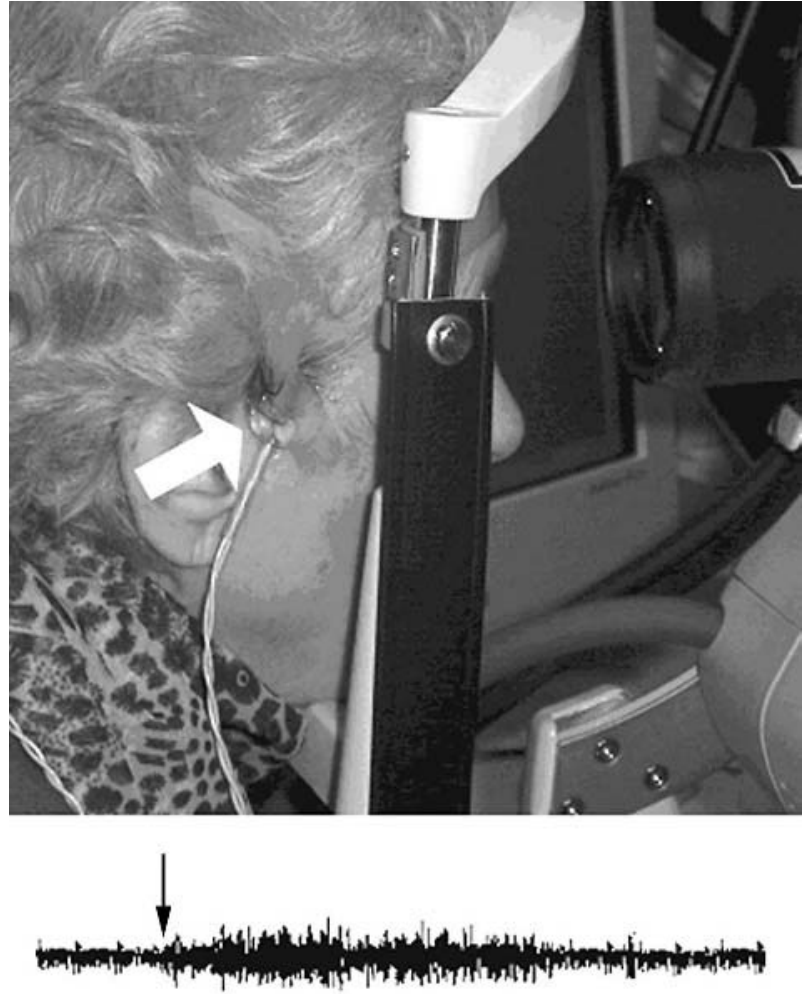

1 second

Figure 1 Top, illustration of the experimental set-up for simultaneous recording of dynamic angiogram and Doppler pulse of the subcutaneous temporal artery (arrow shows the Doppler probe placed in the pretragian area). Bottom, example of Doppler signal of the systolic pulse (arrow shows the beginning of systole).

of the homolateral subcutaneous temporal artery was recorded using a custom made $8 \mathrm{mHz}$ ultrasonic Doppler probe (Stereodop; Promedec, Guer, France) during dynamic videoangiography (fig 1 ). The audio and video signals were then mixed on a miniDV tape recorder (GVD 900E, Sony, Tokyo, Japan). All patients had intraocular pressure less than or equal to $21 \mathrm{~mm} \mathrm{Hg}$ and a blood pressure level less than $160 / 95 \mathrm{mmHg}$ at the time of dynamic angiography

\section{Image analysis}

Dynamic angiograms were reviewed by two of the authors (MP, OG). Analysis of the dynamic angiogram was performed off-line by iteratively playing each patient's sequence, either at normal speed or frame by frame. This allowed us to determine if pulsatile filling of arteries and/or veins was present. The arteriovenous filling time (AVFT), which corresponds to the time elapsing between the appearance of the dye in the central retinal artery and the complete filling of temporal veins, was measured. The PVO velocity was measured manually point by point, and converted to millimetres assuming a horizontal diameter of the disc of $1.8 \mathrm{~mm} .{ }^{12}$ For the patients in which the Doppler signal of the subcutaneous temporal artery had been recorded, the dynamic angiogram was converted to QuickTime files with an iMacDV computer (Apple, Cuppertino, CA, USA). Each frame was then displayed with its corresponding sound intensity spectrum using Adobe Premiere Pro software (Adobe Corporation, Palo Alto, CA, USA).

\section{RESULTS}

The clinical characteristics of the patients are indicated in table 1 .

Three patients (cases 8,9, and 10) had an ischaemic form of CRVO with rubeosis iridis. In the other patients, fluorescein angiography revealed neither rupture of the blood-retinal barrier nor extensive areas of capillary nonperfusion. Cases 1 and 3 had perivenular opacification of the retina, a clinical presentation recently described. ${ }^{13}{ }^{14}$ Cases 1 and 7 had concomitant occlusion of a cilioretinal artery. On dynamic angiograms, all patients had severe impairment of blood flow, with AVFT ranging from 19.8 to 58.3 seconds, the mean (SD) for AVFT in normal subjects being 12.5 (2.3) seconds. ${ }^{10}$ PVO appeared as discrete boli of dye in first order veins at the beginning of the venous phase, progressing towards the disc with a pulsatile motion (see the supplemental video on BJO website (http://www.bjophthalmol.com/ supplemental, and fig 2). PVO originated in all cases from second order veins at their confluences with first order veins, while the flow velocity of first order veins was at its minimum. In two cases ( 3 and 10 ), there was even alternate flow in first order veins, the retrograde flow being observed during and immediately after the onset of PVO (fig 3).

\section{Venous flow variations over the cardiac cycle}

The progression of PVO in first order veins allowed to determine the time of onset and value of $V_{\min }(n=10)$ and $\mathrm{V}_{\max }(\mathrm{n}=6)$. In all patients, $\mathrm{V}_{\min }$ in first order veins was lower than $2 \mathrm{~mm} / \mathrm{s}$ (table 1 ). $V_{\min }$ occurred simultaneously with the onset of PVO, as shown in the example of figure 2. The time of occurrence and value of $\mathrm{V}_{\max }$ with respect to the PVO could be estimated in six of our patients. $V_{\max }$ in first order veins occurred between 0.20 seconds and 0.44 seconds following the onset of PVO (fig 4). Expressed in degrees of

Table 1 Clinical and angiographic data of the patients

\begin{tabular}{|c|c|c|c|c|c|c|c|c|}
\hline Case & Age/sex/side & $\begin{array}{l}\text { Intial } \\
\text { VA }\end{array}$ & Duration & Ischaemic & $\begin{array}{l}\text { AVFT } \\
\text { (seconds) }\end{array}$ & $\begin{array}{l}\text { Vmin } \\
(\mathrm{mm} / \mathrm{s})\end{array}$ & $\begin{array}{l}V \max \\
(\mathrm{mm} / \mathrm{s})\end{array}$ & Final VA \\
\hline 1 & $52 / M / L$ & $20 / 30$ & 1 day & no & 19.8 & $<1$ & 4.7 & $20 / 20$ \\
\hline 2 & $58 / M / R$ & $\mathrm{HM}$ & 15 days & no & 40.1 & $<1$ & ND & ND \\
\hline 3 & $65 / M / L$ & $20 / 200$ & 2 days & no & 48.6 & retrograde & 2.2 & $20 / 25$ \\
\hline 4 & $52 / F / R$ & $20 / 20$ & 20 days & no & 22.3 & 1.8 & 7.1 & $20 / 20$ \\
\hline 5 & $30 / \mathrm{M} / \mathrm{L}$ & $20 / 20$ & 48 days & no & 20.9 & 1.4 & 5.4 & $20 / 20$ \\
\hline 6 & $37 / M / L$ & $20 / 60$ & 3 days & no & 43.4 & $<1$ & 4.5 & $20 / 25$ \\
\hline 7 & $45 / \mathrm{M} / \mathrm{L}$ & $20 / 60$ & 21 days & no & 33 & $<1$ & ND & $20 / 60$ \\
\hline 8 & $60 / \mathrm{M} / \mathrm{L}$ & $\mathrm{HM}$ & 13 months & yes & 58.3 & $<1$ & ND & $\mathrm{HM}$ \\
\hline 9 & $55 / \mathrm{M} / \mathrm{R}$ & CF & 8 days & yes & 44.8 & $<1$ & ND & $20 / 200$ \\
\hline 10 & $60 / M / R$ & $20 / 200$ & 5 days & yes & 21.2 & retrograde & 5 & $\mathrm{CF}$ \\
\hline
\end{tabular}

VA, visual acuity; $\mathrm{HM}$, hand motion; $\mathrm{CF}$, counting fingers; $\mathrm{AVFT}$, arteriovenous filling time; Vmin, minimal velocity in first order veins; Vmax, maximum velocity in first order veins; ND, not determined. 

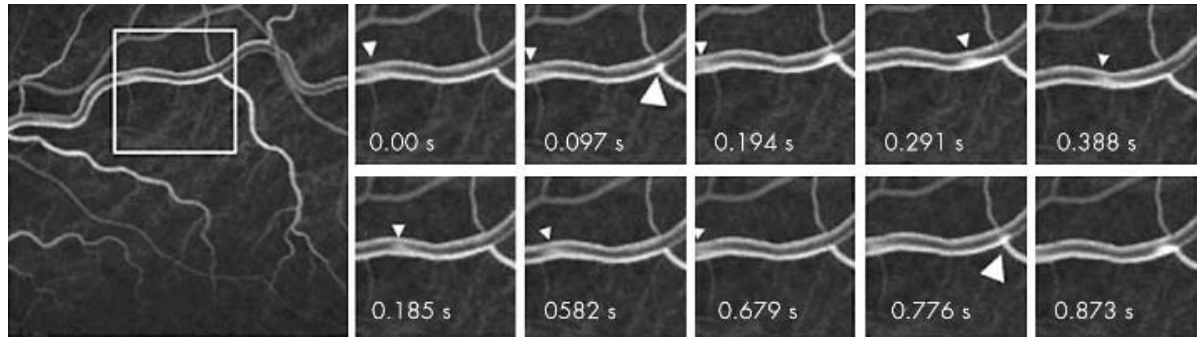

Figure 2 Case 1. Consecutive SLO frame at the arteriovenous phase of dynamic angiography during central retinal vein occlusion. The successive frames on the right are magnification of the area indicated on the left. Note the pulsatile venular outflow (PVO; large arrowheads) which progresses in the superotemporal vein (small arrowheads).

cardiac cycle, this delay ranged from $72^{\circ}$ to $190^{\circ}$. $\mathrm{V}_{\max }$ ranged from 2.2 to $7.1 \mathrm{~mm} / \mathrm{s}$.

\section{Temporal relation between systolic pulse and venous flow}

In five patients (cases $2-4,8$, and 9), the presence of pulsatile filling of arteries allowed an estimation of the delay between arterial systole and PVO onset. This was performed by two different methods. In two patients, the Doppler pulse of the superficial temporal artery was recorded simultaneously with the dynamic angiogram (figs 5 and 6). In three other patients, analysis of the dynamic angiogram showed that the late arterial filling and the early venous filling overlapped during several seconds. It could thus be objectively concluded by direct examination of the video frames that the systolic pulse (that is, the arrival of flow in arteries) occurred either during the same frame or the frame immediately preceding that in which the PVO appeared (fig 7). In all these cases, the delay between arterial systole and PVO was equal to or less than 0.08 seconds.

\section{Clinical outcome}

The mean follow up was 8 months (range 4 weeks12 months). One patient was unavailable for follow up. Five patients had a final visual acuity of 20/40 or better. The three patients with ischaemic CRVO had final visual acuity of less than 20/100. No statistical correlation was found between dynamic angiography features and clinical outcome (data not shown).

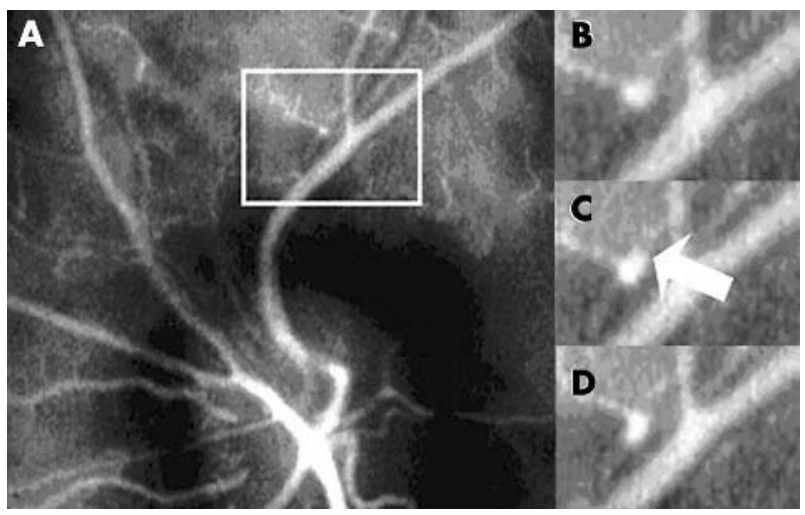

Figure 3 From (A) to (D), consecutive frame from SLO dynamic angiography of case 3. (A) Onset of PVO from a second order vein (square area). (B), (C), and (D) are magnifications of the area indicated in (A). Note the presence of retrograde flow in first order vein (arrow in (C)) immediately after the onset of PVO (frame rate $25 / \mathrm{s}$ ).
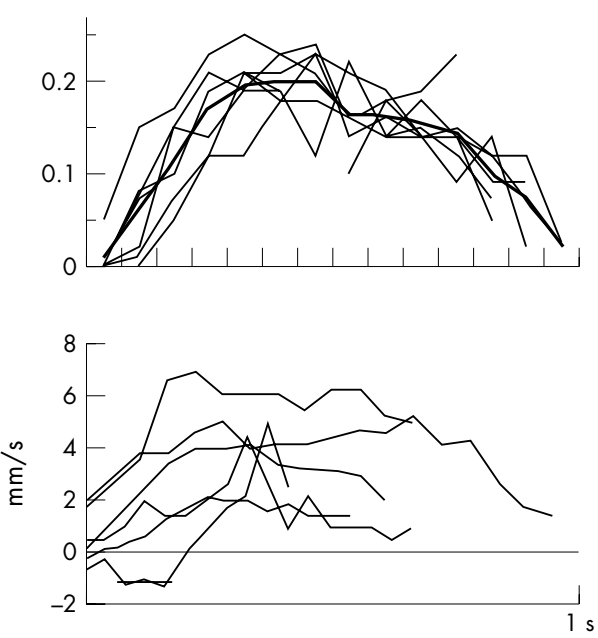

Figure 4 Flow velocity profiles in first order veins over time. Top, case 1. $x$ axis, time in video frame (the origin is the onset of pulsatile venular oufflow); $y$ axis, velocity in $\mathrm{mm} /$ frame. The heavy line is the average of the measurements over seven cardiac cycles. Bottom, superposition of mean velocity profiles of six patients.

\section{DISCUSSION}

In this study, we explored the relation between arterial and venous flow during CRVO, using PVO as an indicator of flow progression. We demonstrate that dynamic angiography is of interest in analysis of venous haemodynamics in cases of severe reduction in blood flow, despite its intrinsic limitations - that is, the sampling rate is limited by the video frame rate. This indeed allowed us to hypothesise that first and second order veins have distinct haemodynamic regimens, which is the cause of the PVO phenomenon.

Firstly, there was evidence of a direct transmission of the systolic arterial pulse through capillaries and post-capillary venules (that is, second order veins). Indeed, the short delay between the systolic pulse and the onset of PVO suggests that it was the systolic pulse itself, transmitted through the capillaries up to venous confluents, that caused the PVO phenomenon. Interestingly, the transmission of the systolic pulse through macular capillaries is present in normal subjects as well, since blue field entoptoscopy demonstrates that the flow in macular capillaries of normal subjects is also pulsatile in synchrony with systole. Thus, this may be a physiological phenomenon, which became prominent in our patients because of the extreme slowing of flow.

Secondly, we show that $\mathrm{V}_{\text {min }}$ in first order veins was simultaneous with the beginning of systole. $V_{\min }$ was even retrograde in two cases. An explanation for this retrograde flow relies on the particular disposition of the retinal vasculature, with a single artery and vein couple embedded in the inextensible optic nerve, ${ }^{15}$ making theoretically 


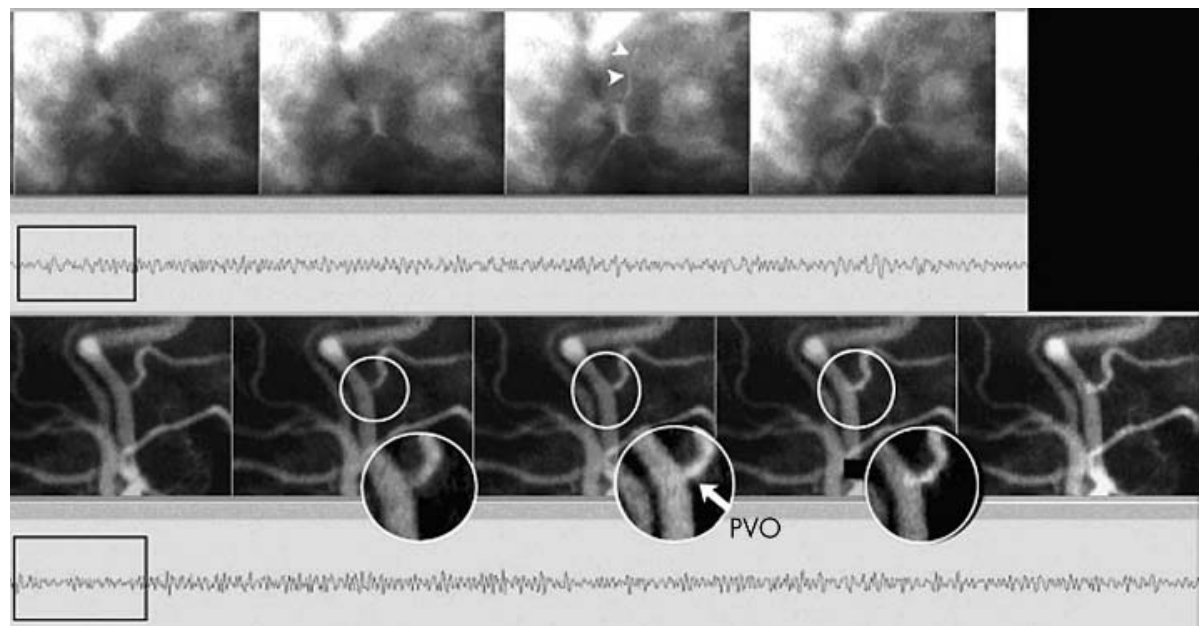

Figure 5 Case 8. Estimation of the delay between systolic pulse and onset of PVO. From left to right, consecutive ICG angiogram frames of the arterial filling (upper images) and of the venous filling (bottom images) overlying the corresponding intensity of the Doppler signal of the homolateral superficial temporal artery. The PVO (arrow in bottom image) appears less than 0.04 seconds following the beginning of the systolic pulse in retinal arteries (arrowhead in upper images).

possible a systolic compression of the central vein by the central artery. Another explanation for the presence of retrograde flow is based on the finding that the IOP and venous flow curves are parallel. ${ }^{11}{ }^{16}$ From this derives the hypothesis that the systolodiastolic variation of IOP modulate the flow in the central retinal vein. It is reasonable to hypothesise that the same effect is present upstream of the central vein-that is, within retinal veins. Indeed, the delay between venous and arterial flow that we found matches the delay between the IOP/venous flow curves and the arterial flow curve reported by Michelson and Harazny. If it is admitted that the intraluminal pressure of retinal veins depends on IOP, then the pressure gradient $(\Delta \mathrm{P})$ between the retrobulbar segment of the central retinal vein and the retinal veins also depends on IOP. Therefore, in the haemodynamic situation of a central vein occlusion, characterised by a low velocity and a high resistance to outflow, ${ }^{16}$ the drop from $\mathrm{IOP}_{\max }$ to $\mathrm{IOP}_{\min }$ and the subsequent drop in intraluminal pressure of first order veins may explain a systolic decrease of $\Delta \mathrm{P}$ and thus a transient retrograde flow. Studies combining laser Doppler velocimetry in arteries and veins to measurement of the IOP curve are needed to verify this hypothesis.

The overall figure that emerges from these findings supports the hypothesis that venous circulation in first order retinal veins is modulated by the arterial systolic impulse transmitted through second order veins, and probably by the IOP curve. We hypothesise that the PVO phenomenon results from the interaction of these two factors. The contribution of other factors such as intraneural compression of the central vein by the central artery remains to be determined.

We found no clear correlation in our series between haemodynamic parameters and the type of CRVO (perfused $v$ non-perfused) or the clinical outcome. All non-perfused patients had $V_{\min }$ of less than $1 \mathrm{~mm} / \mathrm{s}$, compared to five out of seven perfused patients. Even if the small number of patients does not allow reliable statistical analysis, it can be concluded that there is no striking difference between these two groups. In the literature, the relation between retinal haemodynamics and the clinical outcome of CRVO has received relatively little attention. Williamson and Baxter ${ }^{5}$ reported that the presence of non-measurable flow by echo Doppler in the central vein-that is, less than $1 \mathrm{~cm} / \mathrm{s}$, was associated with a worse outcome, with $80 \%$ of these patients developing iris neovascularisation. Comparatively, while most of our patients had a mean flow of less than $1 \mathrm{~cm} / \mathrm{s}$, only three of our patients had an ischaemic form of CRVO requiring laser photocoagulation. One patient with retrograde flow had a good visual outcome. In a previous study, we have shown a case in which after the onset of nonperfusion, retinal haemodynamics improved as assessed by

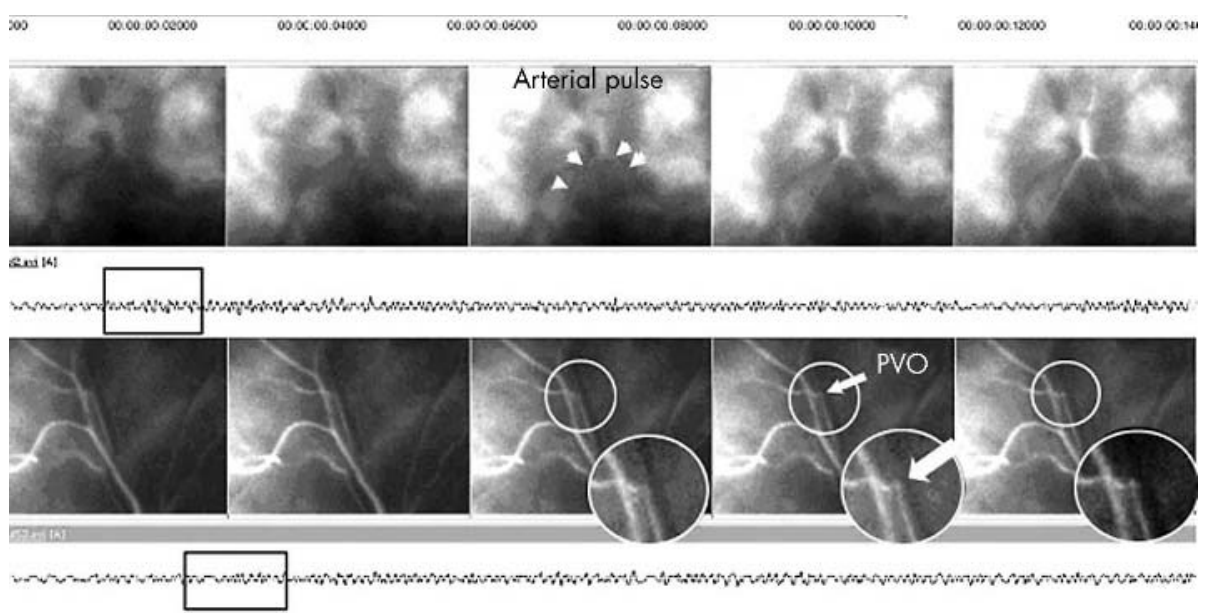

Figure 6 Case 9. Same legend as for figure 5. The PVO (bottom, arrow) appears less than 0.08 seconds after the beginning of the systolic pulse in retinal arteries. 

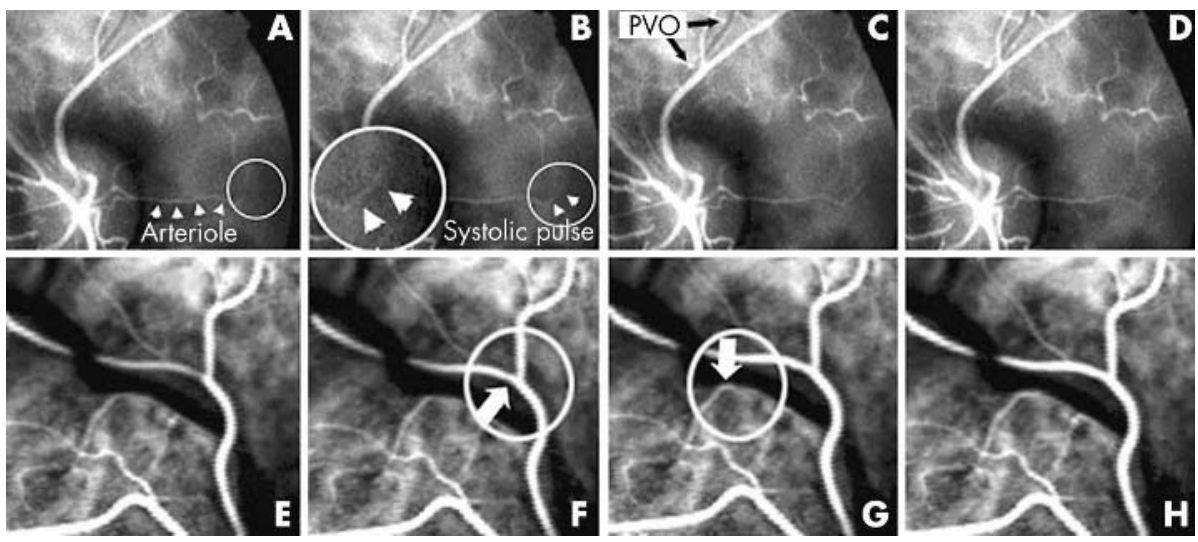

Figure 7 Direct estimation of the delay between systolic retinal arterial pulse and PVO onset in cases $5(A-D)$ and 6 (E-H). From left to right, consecutive images of dynamic ICG angiography. The systolic pulse of retinal arteries ( $B$ and $F$, respectively) is coincident with, or occurs in, the frame immediately preceding the onset of PVO from second order venules ( $C$ and $G$, respectively), indicating a delay of less than 0.08 seconds between the beginning of systole and the onset of PVO.

the disappearance of PVO but non-perfusion persisted. ${ }^{10}$ Overall, this suggests that blood flow decrease is necessary but not sufficient to trigger non-perfusion, which may depend upon other factor(s). A prospective trial is under way in our department to determine if the presence of severely decreased velocity at the acute phase of CRVO implies a worse visual prognosis. This may help to determine the interest of dynamic angiography for the management of patients.

In summary, during CRVO with severe reduction in blood flow, dynamic angiography is a useful tool in the analysis of venous flow. Our results suggest that the PVO phenomenon results from the coexistence of distinct haemodynamic regimens in first and second order veins. We hypothesise that the flow in second order veins is synchronous with arterial flow, while the flow in first order veins is synchronous with the IOP curve and/or under the dependence of an intermittent compression within the optic nerve. Further studies are under way to verify this hypothesis. In this small study, no correlation was found between flow velocity and clinical outcome, suggesting that blood velocity is not the sole factor determining the degree of capillary perfusion.

\section{ACKNOWLEDGEMENTS}

Georges Caputo provided technical help with the Doppler system.

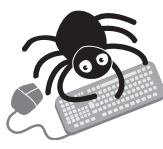

A video showing real time sequence of dynamic angiography at the arteriovenous phase from case 1, showing the pulsatile venular oufflow arising from the confluence of first and second order veins is available on the BJO website (http://www.bjophthalmol.com/ supplemental)

\section{Authors' affiliations}

M Paques, O Genevois, J Sahel, Department of Ophthalmology of the Fondation Ophtalmologique Rothschild, Paris, France
O Baillart, B I Lévy, Departments of Physiology, Paris, France A Gaudric, Ophthalmology of the Hôpital Lariboisière, Paris, France The authors have no proprietary interest in this study.

Ethical approval: The protocol of this study was approved by the ethics committee of the Hopital Saint-Lovis.

\section{REFERENCES}

1 Williamson TH. Central retinal vein occlusion: what's the story? Br J Ophthalmol 1997;81:698-704.

2 Keyser BJ, Flaharty PM, Sergott RC, et al. Color Doppler imaging of arterial blood flow in central retinal vein occlusion. Ophthalmology 1994:101:1357-61.

3 Tranquart F, Arsene S, Giraudeau B, et al. Initial color Doppler findings in retinal vein occlusion. J Clin Ultrasound 2000;28:28-33.

4 Avunduk AM, Dinc H, Kapicioglu Z, et al. Arterial blood flow characteristics in central retinal vein occlusion and effects of panretinal photocoagulation treatment: an investigation by colour Doppler imaging. Br J Ophthalmol 1999;83:50-3

5 Williamson TH, Baxter GM. Central retinal vein occlusion, an investigation by color doppler imaging. Ophthalmology 1994;101:1362-72.

6 Remky A, Wolf S, Knabben H, et al. Perifoveal capillary network in patients with acute central retinal vein occlusion. Ophthalmology 1997; 104:33-7.

7 Schatz H, Fong AC, McDonald HR, et al. Cilioretinal artery occlusion in young adults with central retinal vein occlusion. Ophthalmology 1991;98:594-601.

8 Keyser BJ, Duker JS, Brown GC, et al. Combined central retinal vein occlusion and cilioretinal artery occlusion associated with prolonged retinal arterial filling. Am J Ophthalmol 1994;117:308-13.

9 Brown G, Duker J, Lehman R, et al. Combined central retinal artery and central vein obstruction. Int Ophthalmol 1993;17:9-17.

10 Paques M, Garmyn V, Catier A, et al. Analysis of retinal and choroidal circulation during central retinal vein occlusion with ICG videoangiography. Arch Ophthalmol 2001;119:1781-7.

11 Michelson G, Harazny J. Relationship between ocular pulse pressure and retinal vessel velocities. Ophthalmology 1997;104:664-71.

12 Hellstrom A, Svensson E. Optic disc size and retinal vessel characteristics in healthy children. Acta Ophthalmol Scand 1998;76:260-7.

13 Browning DJ. Patchy ischemic retinal whitening in acute central retinal vein occlusion. Ophthalmology 2002;109:2154-9.

14 Paques M, Gaudric A. Perivenous macular whitening during central retinal vein occlusion. Arch Ophthalmol 2003;121:1488-91.

15 Taylor AW, Sehu W, Williamson TH, et al. Morphometric assessment of the central retinal artery and vein in the optic nerve. Can J Ophthalmol 1993;28:320-4.

16 Michelson G, Harazny J. Increased vascular resistance for venous outflow in central retinal vein occlusion. Ophthalmology 1997;104:659-63. 